Voix et Images

voixetimages

\title{
Entretien avec Micheline Lachance
}

\section{Marilyn Randall et Daniel Vaillancourt}

Volume 26, numéro 3 (78), printemps 2001

Généalogies de la figure du Patriote 1837-1838

URI : https://id.erudit.org/iderudit/201563ar

DOI : https://doi.org/10.7202/201563ar

Aller au sommaire du numéro

\section{Éditeur(s)}

Université du Québec à Montréal

\section{ISSN}

0318-9201 (imprimé)

1705-933X (numérique)

Découvrir la revue

\section{Citer ce document}

Randall, M. \& Vaillancourt, D. (2001). Entretien avec Micheline Lachance. Voix et Images, 26(3), 556-565. https://doi.org/10.7202/201563ar d'utilisation que vous pouvez consulter en ligne.

https://apropos.erudit.org/fr/usagers/politique-dutilisation/ 


\title{
Entretien avec Micheline Lachance
}

\author{
Marilyn Randall et Daniel Vaillancourt, \\ University of Western Ontario
}

Micheline Lachance, l'auteure des deux tomes du Roman de Julie Papineau (Québec Amérique, 1995, 1998), a répondu par l'écrit à une série de questions que nous lui avons suggérées. Les réponses sont présentées sans modification. Cet entretien n'a donc pas constitué un échange d'idées, mais une occasion de sonder l'auteure sur son rapport à son roman, à l'écriture de l'bistoire, aux Rébellions et à Julie Papineau, "femme patriote».

\section{Sur les Rébellions}

V. et I. : Comment expliquez-vous l'intérêt croissant pour la Rébellion et ses personnages?

M. L. : L'engouement actuel pour l'histoire, avant un grand " $\mathrm{H}$ ", n'est pas propre au Québec. Il est tout aussi perceptible ailleurs. Il y a une dizaine d'années, dans toute l'Amérique, l'intérêt se portait sur la saga des autochtones à l'arrivée des Blancs.

Vient un moment dans la vie où l'on éprouve le besoin de savoir d'où l'on vient, quand cela ne serait que pour décider où l'on s'en va. La question est particulièrement percutante au Québec qui jongle avec son avenir depuis longtemps. Si la Rébellion suscite un intérêt particulier, c'est qu'il s'agit d'une page bouleversante du passé. La défaite des Plaines d'Abraham éveille les mêmes émotions. Nul doute que la déportation des Acadiens et la pendaison de Louis Riel passionneraient tout autant le grand public, si les créateurs faisaient revivre ces événements sur un mode dramatique.

V. et I. : Comment en êtes-vous venue à vous intéresser à cette période de l'histoire québécoise?

M. L. : J'écrivais la biographie du cardinal Paul-Émile Léger. Lorsqu'il m'a parlé de son ancêtre patriote, Luc-Hyacinthe Masson, j'ai voulu en savoir plus sur ses activités pendant les troubles de 1837-1838. J'ai alors lu plusieurs ouvrages, dont le Papineau de Robert Rumilly. L'historien publie des extraits de la correspondance de Louis-Joseph Papineau et de sa 
femme Julie. J'ai été intriguée par les propos de celle-ci, qui s'exprimait avec véhémence sur la politique, s'enflammait même. Des mots comme indignation, violence, justice apparaissaient sous sa plume.

Bien naïvement, je croyais que les femmes du $\mathrm{XIX}^{\mathrm{e}}$ siècle étaient des épouses dociles et silencieuses, obnubilées par leurs curés qui prêchaient à des "convaincues" que la. désobéissance civile menait directement en enfer et qui freinaient leurs maris dans leur lutte. J'ai découvert, au contraire, une femme qui affichait ses opinions politiques avec cran et poussait son mari dans l'arène. J'ai poursuivi mes recherches aux Archives nationales du Québec, où j'ai scruté à la loupe - littéralement - les papiers Papineau.

Au départ, j'ai songé à écrire sa biographie. Mais Julie n'est pas une héroïne traditionnelle, de celles à qui l'on consacre ce genre d'ouvrage. Elle n'a fondé ni école, ni hôpital, comme Marguerite Bourgeois ou Jeanne Mance, et ne s'est pas battue contre les Iroquois, comme Madeleine de Verchères. Cependant, elle m'apparaissait comme un merveilleux témoin d'une page bouleversante de notre histoire: la Rébellion de 1837. Elle serait donc les yeux me permettant de faire revivre, dans un roman, des événements qui ont marqué le destin des Québécois.

En toile de fond, il y a la pire crise économique qu'a jamais connu le Bas-Canada et l'épidémie de choléra qui va décimer des familles entières. La trame du roman, c'est la montée du sentiment nationaliste exacerbé par l'attitude hautaine des marchands anglais qui contrôlent les finances. Jusque-là, les patriotes avaient emprunté des chemins démocratiques pour réclamer justice. Mais la révolte gronde. Elle est déclenchée par la fusillade de la Place d'Armes, où trois patriotes sont tués par des soldats de l'armée britannique. À l'avant-scène, Julie Papinèau, une femme qui se retrouve bien malgré elle au cour de la mêlée.

V. et I.: Vous dites avoir tout lu sur la vie au Bas-Canada, au XIX ${ }^{\mathrm{e}}$ siècle. Cette lecture vous a-t-elle réservé d'autres surprises?

M. L.: Oui, d'autant plus que cet épisode de notre histoire est méconnu, nos programmes d'enseignement l'ayant trop souvent escamoté. Ce qui m'a renversée, c'est l'ampleur des représailles, après la Rébellion et l'Insurrection. Elles ont été cruelles et disproportionnées: viols, vols, pillage et incendie des biens appartenant à des citoyens qui n'avaient rien eu à voir avec les événements, emprisonnements injustifiés, procès des accusés dans une langue qu'ils ne comprenaient pas, pendaisons sadiques...

J'ai découvert en outre qu'on véhicule des faussetés sur le sujet. Par exemple, la Rébellion n'a pas été une guerre de races, comme l'a prétendu faussement lord Durham, mais bien une lutte pour la démocratie, comme celle que les Américains venaient de gagner contre l'Angleterre. Ce n'était pas un conflit opposant les Anglais aux Français puisque, dans le Haut-Canada, une rébellion semblable a eu lieu, au même moment, et 
elle portait sur les mêmes griefs. Il faut voir dans ce soulèvement la réaction d'une colonie exaspérée contre les abus et les excès de la mèrepatrie.

Peut-on établir un parallèle entre cette révolte et les revendications autonomistes des Québécois d'aujourd'hui? Les comparaisons sont toujours boiteuses. Les patriotes réclamaient le gouvernement responsable et le contrôle des deniers publics, ce qui est acquis aujourd'hui. N'empêche... En lisant les discours autonomistes du temps, on a parfois l'impression d'entendre les politiciens actuels. Pendant que j'écrivais ce livre, mon mari, Pierre Godin, préparait la biographie de René Lévesque. Au dîner, nous avions l'impression que nos deux héros étaient assis à chaque bout de la table. Et souvent, les opinions de l'un pouvaient être attribuées à l'autre.

V. et I. : Avez-vous fait des liens entre la Rébellion et la Crise d'octobre?

M. L.: On ne peut pas comparer la Crise d'octobre avec la Rébellion. Les felquistes, une poignée d'hommes, se sont livrés à des activités criminelles. Les patriotes, qui représentaient une large partie de la population, ont choisi la voie démocratique pour réclamer justice. Leurs griefs, connus sous le nom de 92 résolutions, ont été acheminés à Londres par les canaux officiels. Et si les choses se sont gâtées par la suite, il serait malhonnête de n'en blâmer que les Canadiens. Les plus éminents historiens ont reconnu que Londres se livrait à des abus criants au Canada.

Il ne faut pas oublier, en outre, que c'est l'armée britannique qui a attaqué les patriotes retranchés à Saint-Denis, après que des mandats d'arrêt aient été émis contre ses chefs. Les Canadiens se sont défendus avec des fourches et de vieux fusils datant du régime français contre la puissante armée britannique. Les historiens reconnaissent aussi que, dans les bagarres opposant les Fils de la liberté aux jeunes Anglais du Doric club, l'armée ne s'est pas montrée impartiale.

V. et I. : Selon vous, quel rôle jouent les Rébellions dans la formation du Québec contemporain?

M. L. : La défaite des Plaines d'Abraham, comme l'échec de la Rébellion et de l'Insurrection de 1837-1838, ont certainement contribué à forger chez les Québécois un certain défaitisme. Mais cette longue habitude de résistance à l'occupant explique peut-être leur détermination à être reconnus comme un peuple distinct. Tant il est vrai que les peuples refusent de mourir... même trois cents ans après avoir été conquis.

\section{Sur l'écriture historique et romanesque}

V. et I. : Pourriez-vous nous indiquer avec plus de précision le type de libertés qu'il fallait prendre pour concevoir un roman à partir des documents historiques? 
M. L. : En écrivant ce livre, j'avais le souci de raconter dans un roman l'histoire vraie de Julie Papineau. Cependant, rien ne m'y obligeait. Par définition, la formule romanesque permet toutes les libertés.

C'est le choix que j'ai fait et je m'y suis tenue. Je n'ai inventé ni personnages, ni événements. Les documents d'archives me permettaient de reconstituer la vie de Julie et des siens le plus rigoureusement possible, comme je l'aurais fait dans une biographie. Mais je voulais aussi recréer le climat de l'époque et présenter des personnages en chair et en os, c'està-dire avec leurs émotions: amour, haine, rancune, déception, jalousie... Il m'importait qu'on les voit manger, pleurer, s'affronter, voyager, ce que la biographie ne permet pas. Je voulais qu'on sente le froid, la peur, le train-train quotidien. Je devais donc dramatiser les événements, comme le fait le cinéaste qui braque sa caméra sur la pluie qui tombe ou sur un couple en pleine querelle de ménage.

J'ai pris quelques libertés afin d'apporter un complément d'information mais en m'assurant de ne trahir ni la vérité historique, ni la pensée des personnages. Un exemple: la pendaison du marchand Dewey, au palais de Justice de Montréal. Je n'ai pas la preuve que Papineau y a amené ses fils. Je sais cependant que, ce jour-là, lui et sa famille étaient à Montréal, que son voisin Jacques Viger y a assisté, de même que son ami, le docteur Robert Nelson, qui a constaté le décès. Comme les pendaisons attiraient tout le faubourg, j'ai décidé d'y amener Amédée et Lactance, ce qui m'a permis de montrer la sensibilité excessive de Lactance, et ainsi préparer le lecteur aux troubles psychiatriques qui affligeront bientôt le jeune homme.

Autre exemple: la fusillade de la Place d'Armes, en juin 1832. C'était jour d'élection et le bureau de vote était situé à côté de l'église. Julie revenait-elle de prier ou de voter? Les deux sont plausibles puisqu'elle était aussi pieuse que patriote. Comme sa révolte est née de cet événement sanglant - trois patriotes tués par des soldats anglais - , ce dont ses lettres témoignent, je l'ai envoyée voter. Il s'agit d'une petite complicité féminine, les femmes mariées et propriétaires n'ayant eu le droit de vote qu'entre 1798 et 1834 . Julie n'a donc pas dû voter souvent... d'autant plus que Louis-Joseph était réticent au vote des femmes.

Dernier exemple: en décembre 1837, le journal Le Canadien a annoncé la fausse nouvelle de la mort de Julie. C'est vrai. J'ignore cependant si, comme je le raconte, Lactance a fait le voyage depuis Saint-Hyacinthe où il était pensionnaire jusqu'à Verchères, pour aller au chevet de sa mère malade. C'est probable car il l'adorait. En refaisant avec lui le trajet, j'ai pu montrer aux lecteurs les villages incendiés de Saint-Charles et Saint-Denis et les villageois apeurés, au lendemäin des batailles sanglantes.

En ce qui concerne les dialogues, je les ai conçus à partir des propos qu'ont réellement tenus les personnages, surtout lorsqu'ils expriment leurs 
opinions politiques. Quand, dans les années quarante, Papineau et LaFontaine discutent du Canada-uni que l'un désapprouve et que l'autre souhaite, je me suis assuree de mettre dans leurs bouches des paroles qui reflétaient leur pensée. Pour bâtir ce dialogue et bien d'autres, j'ai donc puisé dans leurs discours respectifs et leur correspondance. Il n'est pas certain qu'ils aient eu cette discussion à table, en sirotant du vin. Mais une chose est sûre: ce que je leur fais dire, ils l'ont réellement dit.

Devant deux versions crédibles, laquelle fallait-il choisir? Comme l'historien, le romancier, placé devant ce dilemme, doit trancher au meilleur de sa connaissance. Prenons l'exemple du présumé complot du général Colborne pour amener les patriotes à prendre les armes. L'historien Gérard Filteau affirme que les patriotes n'ont pas voulu la lutte armée. Il rappelle que Colborne, surnommé le vieux brulôt, a fait circuler le bruit qu'ils étaient armés jusqu'aux dents, ce qui était faux, et qu'avec son ami le journaliste Adam Thom, il a organisé des rendez-vous clandestins avec les bureaucrates pour élaborer une stratégie afin d'amener les patriotes à se battre pour ensuite les arrêter. Robert Rumilly, par contre, croit que ceux-ci se préparaient à la lutte armée. À preuve les discours enflammés des chefs Papineau et Wolfred Nelson. Ce dernier n'a-t-il pas lancé du haut d'une tribune que le moment était venu de "fondre nos ustentiles pour en faire des balles"? Dans mon récit, j'ai exposé les faits rapportés par l'un et l'autre historiens et j'ai puisé dans la correspondance de mes personnages pour proposer leur propre version des événements.

V. et I.: Si vous deviez réécrire Julie sous forme de biographie, quels changements au niveau du contenu s'imposeraient? Quelles conclusions voudriez-vous amener le lecteur à tirer?

M. L.: Si j'écrivais une biographie de Julie Papineau, le contenu resterait le même. Seule la forme serait modifiée. J'éliminerais forcément les mises en scène et une partie des dialogues. La différence se ferait aussi sentir dans l'écriture nécessairement plus froide, plus objective. Et naturellement, j'indiquerais mes sources, ce qu'on ne peut pas faire dans un roman.

J'aimerais que mes lecteurs aient, après avoir lu cette biographie, une idée plus juste des Rébellions trop souvent racontées de façon biaisée ou incomplète. Je voudrais qu'ils découvrent l'angoisse et l'émoi que les troubles ont suscités chez les Canadiens du Bas-Canada et les répercussions de ces événements dans les siècles suivants. Enfin, je souhaiterais qu'ils aient une meilleure connaissance de la vie des femmes au xix ${ }^{t}$ siècle, qu'on a trop souvent "oubliées" dans les récits. Ces conclusions sont les mêmes que celles que je propose aux lecteurs du Roman de Julie Papineau.

V. et I.: Étant donné les recherches historiques approfondies auxquelles vous vous êtes livrée pour rédiger vos romans, à quel type de réception vous attendiez-vous? 
M. L.: Je n'avais aucune attente précise. J'ai écrit ce roman parce que le destin de Julie me passionnait et que j'avais envie de le raconter. Je souhaitais ardemment que les gens prennent autant de plaisir à le lire que j'en ai eu à l'écrire.

Il m'a fallu dix ans de recherche et d'écriture pour compléter les deux tomes du Roman de Julie Papineau. J'avais déjà signé deux biographies (Le frère André et Le cardinal Léger) et j'avais envie de tenter l'expérience romanesque en recréant la vie au $\mathrm{xIX}^{e}$ siècle. Je voulais combiner la rigueur historique et l'écriture littéraire et je m’y suis appliquée.

Les historiens ont accueilli avec respect et sympathie mes deux ouvrages, ce que j'ai grandement apprécié. L'un d'eux, éminent professeur à l'Université de Montréal, m'a dit: "Vous avez mis de la chair sur des personnages que je m'efforce de présenter d'une manière scientifique. "Et un autre: "Vous rejoignez des gens que je ne peux pas atteindre."

V. et I. : Devrait-on les considérer comme une contribution importante à la recherche historique?

M. L. : Ce n'est pas à moi de répondre à cette question. On m'attribue habituellement le mérite d'avoir sorti Julie Papineau des boules à mites. Avant mes livres, le grand public n'avait à peu près jamais entendu parler d'elle. Depuis, une exposition lui a été consacrée dans un musée de Québec, sa correspondance a été publiée, un projet de télésérie est à l'étude et des universités se penchent sur son cas. Enfin, les sociétés historiques et les bibliothèques me demandent constamment des conférences sur elle et sur les femmes de son époque.

V. et I.: Dans une entrevue dans Le Devoir, vous notez que la liaison entre Papineau et Marcella Dowling, racontée dans le roman, est inscrite "noir sur blanc" dans la correspondance aux archives et, pourtant, que "certains croiront que c'est une partie de romance que j'ai inventée de toutes pièces". Pour un auteur qui veut relater les faits documentés, cette ambiguiité ne serait-elle pas l'un des dangers de l'histoire romancée?

M. L. : À ma connaissance, cet amour, peut-être purement platonique, n'a jamais été mentionné dans les ouvrages d'historiens. Il s'agit donc d'une contribution à l'histoire, dans la mesure où il explique en partie le comportement désinvolte de Papineau à Paris, alors que plusieurs de ses compatriotes l'attendaient désespérément à Montréal. Je ne vois pas quel danger il y aurait à le relater dans un roman. Je m'étonne cependant que les biographes de Papineau ne l'aient pas mentionné.

Il est vrai que certains pourraient croire que j'ai inventé cette liaison, qui aurait sa place dans un roman d'amour traditionnel. Ne dit-on pas parfois d'un fait véridique que c'est un "vrai roman"? Même romancée, une histoire peut être rapportée dans toute sa vérité. N'est-ce pas ce que fait le cinéma? 
V. et I.: Quelle importance attachez-vous à ce que le roman soit lu comme histoire?

M. L. : L'histoire me passionne depuis mon tout jeune âge, et j'avais envie de partager cette passion avec le plus large public possible. Les gens lisent dans le métro ou le soir avant de s'endormir. Pourquoi ne pas leur proposer une lecture de détente qui soit instructive? Dans les salons du livre, les lectrices qui me parlent de Julie me disent qu'elles se reconnaissent en cette femme attachante, jalouse, mère poule... Elles admirent son courage dans l'adversité et apprécient le fait qu'elle n'ait pas la langue dans sa poche. Ensuite, elles me questionnent sur la Rébellion, me confient qu'elles iront visiter le manoir Papineau à Montebello "pour en savoir plus" et qu'elles peuvent maintenant expliquer à leurs enfants ce qui s'est passé jadis à la Place d'Armes, au marché Bonsecours ou ailleurs dans le Vieux Montréal. Ma façon de transmettre l'histoire n'est peut-être pas conventionnelle mais elle a le mérite de captiver des gens qui autrement ne s'y intéresseraient peut-être pas.

V. et I.: Outre la correspondance de Julie, celle du grand-père, ainsi que le journal du fils Amédée, y a-t-il d'autres documents qui ont informé de façon essentielle votre roman?

M. L. : J'ai trouvé de précieux renseignements dans la Saberdache de Jacques Viger, l'un des historiens les plus éclairés de son temps. Ses notes portent sur la vie quotidienne dans le faubourg, sur les voyages entre Montréal et Québec et sur la politique. Comme il était le voisin des Papineau, il m'a fourni une foule de détails utiles. Le journal manuscrit de Lactance Papineau m'en a appris beaucoup sur la vie des Papineau à Paris. Les travaux des historiens, en particulier ceux de Fernand Ouellet sur la vie sociale et économique, m'ont permis de mieux saisir l'époque. La correspondance de monseigneur Jean-Jacques Lartigue m'a éclairée sur le rôle de l'Église pendant les troubles. Enfin, les journaux, notamment $L a$ Minerve et Le Canadien, relatent la vie politique au Bas-Canada et en commentent les soubresauts.

C'est souvent en faisant des recoupements entre les renseignements glanés dans des documents différents que j'ai fini par saisir ce qui s'est réellement passé, car les descendants des Papineau ont effectué un certain nombre de coupures au ciseau dans les lettres de leurs illustres ancêtres.

V. et I. : Comme vous l'indiquez, Julie Papineau a la réputation d'être "déprimée, mélancolique, plaignarde..." Aviez-vous comme but de "réhabiliter" sa réputation?

M. L. : Non. Dans sa biographie intitulée Julie Bruneau, un cas de mélancolie janséniste (Presses de l'Université Laval, 1961), l'historien Fernand Ouellet la décrit comme étant mélancolique, maladive, autoritaire, scrupuleuse, pessimiste, etc. C'est ainsi que la réputation de Julie est née. Après 
avoir lu les mêmes documents d'archives que monsieur Ouellet, j'en suis arrivée à une toute autre conclusion. Julie m'est apparue délurée, sensible, oui, mais ô combien déterminée. Comment expliquer nos divergences? Nous avons interprété différemment certains faits et les mots pour les dire.

Pour ne retenir qu'un exemple, disons que Ouellet affirme que Julie se plaignait sans cesse de ses maladies. Or, en lisant la correspondance de ses contemporains, j'ai pu constater que tous, hommes et femmes, donnent à leurs correspondants des nouvelles de leur santé (comme nous le faisons au téléphone), avant de passer à un autre sujet. De plus, lorsque Julie écrit " J'irai vous voir quand j'aurai terminé ma maladie", elle fait référence à sa grossesse qui achève et non à quelque mal imaginaire. Comme elle a accouché dix fois en quinze ans, il n'est pas étonnant qu'elle ait été "malade" souvent.

Quant à sa tendance à la mélancolie, elle n'a rien de surprenant chez une femme qui a traversé des épreuves aussi cruelles: la mort de cinq enfants en bas âge, la mise à prix de la tête de son mari, l'emprisonnement de ses amis, l'exil forcé, la folie d'un de ses fils, etc. Je n'ai pas cherché à la réhabiliter. Je l'ai peinte telle que je l'ai vue, avec ses emportements, sa sensibilité à fleur de peau et sa passion pour la politique. Mais je dois reconnaître qu'après lui avoir consacré plus de mille pages, elle est bien vengée du piètre portrait que l'Histoire avait gardé d'elle.

V. et I.: Dans un article publié dans Liberté en 1965, Charlotte Savary érige Julie comme "symbole d'un peuple déçu" et propose qu'il y aurait lieu de douter que Julie ait été une "épouse comblée". Qu'en pensezvous?

M. L. : Julie a été souvent malheureuse et pour cause. Jeune, elle avait tout pour mener la vie douillette des grandes bourgeoises de son temps. Mais le destin en a décidé autrement. La politique a tenu son mari loin d'elle pendant des mois, la forçant à une solitude non désirée. Plus tard, elle a vécu en exil, loin de ses proches, avec un homme amer et déprimé, qui ne se pardonnait pas d'avoir entraîné son peuple dans une aventure sanglante qu'il n'avait ni prévue ni souhaitée. Elle lui en a voulu, ensuite, de priver leurs enfants d'un père, en s'obstinant à rester en Europe, au lieu de rentrer au pays avec sa famille.

Nul doute, Julie était follement amoureuse de Louis-Joseph et elle l'a épaulé dans ses projets et consolé dans ses revers. Néanmoins, son mari l'a déçue. À la fin de sa vie, elle avait la nostalgie du temps où il était le chef et elle insista fortement pour qu'il reprenne sa place dans l'arène politique. Lui, il ne rêvait plus que d'une vie tranquille, entouré de sa famille et de ses livres, dans son manoir de Montebello qu'il avait voulu grandiose pour plaire à sa femme. Mais Julie ne s'est jamais habituée à y vivre. 
V. et I. : Entre le portrait de Julie et sa fille (Plamondon, 1836) et celui de la couverture du premier tome du roman (Tiffet, 1995), portrait inspiré du premier, il y a lieu de noter un léger embellissement de la représentation de Julie.

M. L. : J'ai écrit un roman et non une biographie, comme le titre du livre l'indique. Pour qu'il n'y ait pas d'ambiguïté à ce sujet, j'ai décidé de présenter mon héroïne sous des traits fictifs, comme c'est toujours le cas lorsqu'il s'agit d'un ouvrage romanesque. Ma Julie a été peinte par l'artiste Tiffet, telle je l'ai imaginée à partir des écrits de ses proches et en m'inspirant du portrait flatteur que Jacques Viger trace de sa "belle voisine". Pour la même raison, afin d'éviter qu'il y ait confusion dans les genres, j'ai intitulé mes livres Le Roman de Julie Papineau, plutôt que simplement Julie Papineau.

\section{Sur la figure de la « femme Patriote»}

V. et I. : Julie Papineau est exceptionnelle sous bien des angles. Pouvezvous nous proposer d'autres figures de femmes Patriotes?

M. L. : C'est l'époque qui est exceptionnelle. Je ne pense pas que Julie le soit tellement plus qu'une autre femme de son temps. Grâce à ses talents d'épistolière, nous la connaissons mieux que d'autres.

Des Julie Papineau, il y en a plus d'une dans l'histoire des patriotes. Qu'on pense à Henriette de Lorimier, dont le mari a été pendu après un procès bâclé; à Adèle LaFontaine et Émilie Gamelin, qui ont soigné les patriotes emprisonnés au Pied-du Courant, en dépit de la présence menaçante de l'armée britannique; à Émilie Boileau, qui a tenu chez elle à Chambly des assemblées politiques, au risque de voir sa maison incendiée; à Marie-Rosalie Dessaulles, qui abritait et nourrissait les pauvres et les malades, dans son manoir à Saint-Hyacinthe... Il y a aussi les patriotes sans nom qui se sont montrées courageuses pendant les troubles, notamment celles qui ont été chassées de leur maison et qui se sont enfuies à moitié nues dans la forêt, leurs enfants affolés à leurs trousses; celles qui ont soigné les blessés, caché des rebelles, fabriqué des munitions pour défendre leurs villages. Enfin, il y a les femmes de Saint-Eustache qui ont cousu le premier drapeau canadien-français. Elles ont toutes existé. Il suffirait peut-être de vouloir retracer certaines d'entre elles et ainsi leur redonner un nom.

V. et I. : Selon vos connaissances des archives, peut-on espérer mieux connaître ces femmes par des moyens documentaires?

M. L. : Récemment, j'ai aussi écrit trois nouvelles dont les héroïnes sont des femmes à peine connues et seulement à cause de leurs maris ou de leurs pères: Catherine Delzenne, épouse du docteur Pierre de Sales Laterrière, Henriette Cadieux, veuve de Chevalier de Lorimier, et Azélie Papi- 
neau, fille de Louis-Joseph Papineau et épouse du peintre Napoléon Bourassa. J'aurais pu écrire des romans entiers à leur sujet, tant la documentation est impressionnante. Encore fallait-il chercher...

J'ai en tête deux ou trois autres portraits de femmes qui occuperont mon temps au cours des prochaines années. Des femmes ayant réellement vécues mais qui sont demeurées dans l'ombre. Je les ressusciterai dans des romans, car j'adore ce genre littéraire, et le succès du Roman de Julie Papineau me donne à croire que les lecteurs l'apprécient aussi.

V. et I. : Dans la "note de l'auteur", vous dites vous être posé la question: "Comment une femme de son temps comme Julie osait-elle juger les hommes politiques, accuser les gouverneurs anglais d'injustice et aller jusqu'à suggérer que les Canadiens devraient peut-être prendre les armes pour obtenir justice?" Comment répondez-vous à cette question?

M. L. : Cette question intrigante est à l'origine de mon projet d'écrire Le roman de Julie Papineau. Les manuels d'histoire portant sur le $\mathrm{xIX}^{\mathrm{e}}$ siècle nous ont transmis l'image de femmes dociles et résignées. D'où ma surprise d'en découvrir une qui n'avait pas froid aux yeux. Après dix ans de recherche, je suis maintenant convaincue que les femmes de cette époque, bourgeoises et prolétaires, étaient beaucoup plus délurées qu'on l'imagine.

Bon nombre d'entre elles étaient courageuses, débrouillardes et énergiques. Elles ont souvent assumé la responsabilité de deux, parfois trois générations, ont contribué à faire vivre leurs familles et avaient à cour l'éducation des leurs. Ce n'était pas une mince tâche. On aurait tort, en outre, de sous-estimer leur influence sur le destin politique de leurs contemporains.

Julie est sans conteste une femme de son temps. Née à peine 35 ans après la conquête, elle a vécu les retombées de ce drame qui a bouleversé sa famille et transformé la vie de son milieu. Fille d'un député, elle a grandi entouré d'hommes politiques, a épousé le plus prestigieux d'entre eux et a fréquenté les personnalités influentes du $\mathrm{xIX}^{\mathrm{e}}$ siècle. De plus, elle a vécu des années troubles, ponctuées de violence, dans un pays sous domination anglaise. Elle était fière de ses origines françaises et de sa patrie. Lucide aussi, quant au comportement des marchands et bureaucrates anglais à l'égard des Canadiens. Tout cela explique sans doute sa passion pour la politique et son besoin de s'exprimer.

Il n'est pas surprenant qu'elle ait influencé son mari, au moment de prendre des décisions cruciales. Au risque de surprendre, je concluerais en disant qu'elle m’a souvent semblé plus déterminée que lui. 